\title{
Las TIC en el proceso de enseñanza-aprendizaje del curso de Física
}

\section{The TIC in the teaching-learning process of the Physics Course}

\section{Referencia del artículo}

\author{
Mario Israel Argueta Dardón \\ Facultad Humanidades \\ Universidad de San Carlos de Guatemala \\ fisicamairgd@gmail.com
}

Argueta, M. (2020) Las TIC en el proceso de enseñanza-aprendizaje del curso de Física. Revista Guatemalteca de Educación Superior, 1(1), 112-120. DOI: https://doi.org/10.46954/revistadusac.v1i1.12

Recibido 04/10/ 2020

Revisado 10/10/2020

Aceptado 30/10/2020

\section{Resumen}

La presente publicación consiste en mostrar la importancia que tienen las TIC en el proceso de Enseñanza Aprendizaje, dar a conocer el nivel de competencia académica, estilos de aprendizaje y estrategias a utilizar según los resultados, empleando una metodología descriptiva y transversal a través de una prueba diagnóstica del curso.

Dado el alto nivel de repitencia en el curso de Física de la Facultad de Ciencias Médicas, este trabajo investiga los posibles indicadores que inciden en el problema que aqueja a la mayoría de los jóvenes ya que el porcentaje de reprobación es superior al $50 \%$. Se estima que los estudiantes no se adaptan rápidamente al cambio entre la educación media y universitaria y, tienen dificultades para organizar su tiempo. Los alumnos, además, carecen de técnicas para estudiar, presentan un bajo nivel de comprensión lectora y la carrera de nivel intermedio no es acorde al perfil de ingreso. 
De acuerdo con los resultados del trabajo de campo, se obtuvo que un $73 \%$ de los estudiantes reprobaron la prueba práctica en el área numérica. La prueba de Estilos de Aprendizaje del Modelo HoneyAlonso mostró que el $4 \%$ de los estudiantes cuentan con un aprendizaje teórico, el $15 \%$ con un aprendizaje activo, el 58\% con un aprendizaje reflexivo y un $23 \%$ con un aprendizaje pragmático.

Por ello, se realizó una propuesta didáctica de aplicación para el fortalecimiento del uso de recursos tecnológicos [TIC] y el mejoramiento del proceso de enseñanza aprendizaje de los estudiantes evaluados.

\section{Abstract}

This publication consists of showing the importance of TIC in the Teaching-Learning process, making known the level of academic competence, learning styles and strategies to be used according to the results, using a descriptive and transversal methodology through a test diagnosis of the course.

Given the high level of repetition in the Physics course of the Faculty of Medical Sciences, this work investigates the possible indicators that affect the problem that affects most young people since the failure rate is higher than $50 \%$. It is estimated that students do not adapt quickly to the change between high school and university and have difficulties to organize their time. The students also lack study techniques, have a low level of reading comprehension and the intermediate level career is not consistent with the entry profile.

According to the results of the field work, it was obtained that $73 \%$ of the students failed the practical test in the numerical area. The HoneyAlonso Model Learning Styles test showed that $4 \%$ of students have theoretical learning, 15\% active learning, 58\% reflective learning and $23 \%$ pragmatic learning.

Therefore, a didactic application proposal was made to strengthen the use of technological resources [ICT] and to improve the teachinglearning process of the evaluated students.
Palabras clave

Enseñanza, aprendizaje, tecnología, curso, física.
Keywords

Teaching, learning, technology, course, physics. 


\section{Introducción}

El modelo pedagógico constructivista se ha posicionado como una de las mejores opciones con las que cuentan los docentes para generar procesos de enseñanza aprendizaje, aplicando la tecnología como una herramienta de trabajo en la generación de aprendizaje significativo. Con base en la especialización docente surge el interés por la implementación de las TIC en el campo de estudio de la Física para los estudiantes que asisten al primer año de la Facultad de Ciencias Médicas de la Universidad de San Carlos de Guatemala.

Inicialmente el trabajo detallará las generalidades del estudio $y$, después, la línea de investigación que corresponde a Tecnologías de la información y redes sociales. El planteamiento del problema aborda el alto nivel de repitencia del curso, superior al 50\% según los resultados tanto de las evaluaciones finales en los últimos dos años, como de las evaluaciones realizadas en la cátedra de física durante el primer semestre del año en curso.

A partir de estos, se realizará una descripción de los factores que inciden en dicha problemática. El estudio de Ríos (2014) señala que el abandono y la repitencia estudiantil son dos problemas importantes, los cuales inciden en la disminución significativa de la eficiencia terminal en las instituciones universitarias y la larga duración de los estudios de pregrado. Esta situación genera mayores costos privados a los estudiantes y gastos extras en las instituciones públicas de educación superior. El problema implica un retraso en la entrada al mercado laboral generando costos sociales y económicos.

Como detalle metodológico, este estudio es de naturaleza descriptiva y transversal. Se considera descriptivo el estudio cuyos datos son utilizados con finalidad puramente de caracterización descriptiva, no enfocados a una presunta relación causa-efecto. El objetivo de los estudios descriptivos es describir características en una/s población/es determinada/s, por lo tanto, son útiles cuando se conoce poco acerca de lo que queremos estudiar y normalmente sirve como inicio de posteriores investigaciones analíticas.

Debemos tener en cuenta que en los estudios descriptivos no analizamos asociación de causalidad, como la relación entre enfermedad (efecto) 
y determinadas características (causas), García (2004). Se consideran transversales los estudios en los que se examinan la relación entre un fenómeno y una serie de variables en una población determinada y en un momento del tiempo, García (2004).

El objetivo general planteado para esta investigación fue identificar factores asociados con el uso y conocimiento de las TIC en el proceso de enseñanza-aprendizaje del estudiante de primer año del curso de Física en la Facultad de Ciencias Médicas. Los objetivos específicos consisten en identificar los estilos de aprendizaje de los estudiantes de Física de primer año de la Facultad de Ciencias Médicas y, realizar un diagnóstico educativo de los conocimientos básicos con el propósito de establecer el nivel de capacidades, propio del perfil de ingreso al curso bajo estudio. Integrado a esto, se diseñó una propuesta didáctica de aplicación de recursos tecnológicos para el mejoramiento del proceso de enseñanza aprendizaje, aplicable a la población estudiantil que cursa la asignatura de Física.

Posteriormente, se realiza la exposición de la fundamentación teórica, que abarca estudios, informes, artículos e investigaciones de actualidad sobre la temática de aprendizaje, estilos de aprendizaje y sus formas de evaluación, así como el tema de tecnologías de la educación, atendiendo a los objetivos planteados. De esta manera, se muestra la relación teórica que tienen los factores del proceso educativo, en particular, la vinculación entre el uso de recursos tecnológicos, estilos de aprendizaje y su pertinencia (Téliz, 2015; Molina, 2012; Escurra, 2011; Canos y Líen, 2015 y Galarza, 2015).

Los resultados y procesamiento de datos se presentan en el tercer apartado. Incluyen los resultados obtenidos de la prueba diagnóstica de conocimiento básico del curso de Física y sus principales hallazgos. Asimismo, se presentan gráficamente los resultados de la evaluación sobre los estilos de aprendizaje de los estudiantes. Estos resultados son los que fundamentan la orientación técnica de la propuesta didáctica elaborada, en cuanto a la composición del número de actividades conforme al estilo de aprendizaje correspondiente, dentro del contexto del curso de Física a estudiantes de Primer año de la Facultad de Ciencias Médicas de la Universidad de San Carlos de Guatemala. 


\section{Materiales y métodos}

El material empleado consiste en la elaboración de una prueba diagnóstica de conocimientos básicos de física y una prueba sobre estilos de aprendizaje, para los estudiantes del primer año de la Facultad de Ciencias Médicas, los cuales conforman una muestra total de 95 estudiantes asignados que corresponden a cuatro secciones como grupo representativo de estudio.

\section{Resultados}

Figura No. 1.

Características Generales de las cuatro secciones evaluadas en forma porcentual

\section{Resultados de Prueba Diagnostica}

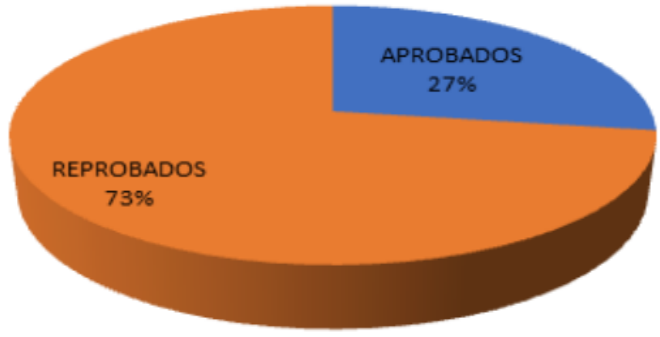

Nota. El grafico representa la evaluación del uso y conocimiento de las TIC en el proceso de enseñanza-aprendizaje del estudiante de primer año del curso de Física en la Facultad de Ciencias Médicas del año 2017 Fuente elaboración propia Argueta, 2019.

De los 95 estudiantes que representan el 100\% de la muestra sólo el $27 \%$ estudiantes aprobaron que son la cuarta parte, mientras que reprobaron la prueba diagnóstica el $73 \%$ de la muestra seleccionada, lo que significa que reprobó la mayoría. En la prueba diagnóstica el promedio de aprobados de la muestra es del $27 \%$; ello se constituye en un indicador de comportamiento negativo respecto del rendimiento de estudiantes de primer año de Ciencias Médicas de la Universidad de San Carlos de Guatemala. Su incidencia en fenómenos de bajo rendimiento académico y repitencia ha sido notoria desde una perspectiva histórica y se constituye en uno de los principales retos de la administración educativa y el ejercicio docente actual. 


\section{Tabla 1.}

Resultados de estilos de aprendizaje.

\begin{tabular}{|c|c|}
\hline Estilo aprendizaje & \% estilos de estilos aprendizaje \\
\hline Activo & $10 \%$ \\
\hline Pragmático & $17 \%$ \\
\hline Teórico & $18 \%$ \\
\hline Reflexivo & $55 \%$ \\
\hline Total & $100 \%$ \\
\hline
\end{tabular}

Nota. esta tabla muestra la evaluación del uso y conocimiento de las TIC en el proceso de enseñanza-aprendizaje del estudiante de primer año del curso de Física en la Facultad de Ciencias Médicas del año 2017.

A partir del trabajo de campo, se puede concluir que la composición de los Estilos de aprendizaje de la población universitaria de primer año, de la Facultad de Ciencias Médicas, el 55\% de estudiantes aprenden de forma reflexiva, sin embargo sólo 15\% aprobaron la evaluación diagnóstica y los 37 restantes reprobaron la prueba, situación que puede estar estrechamente relacionada con el tipo de evaluación que se presenta, puesto que en el caso de los estilos pragmáticos aprobaron solo seis, activo cuatro y teórico uno.

Como características de esta población de estudio, se puede establecer que este tipo de estudiantes consideran experiencias desde diferentes perspectivas, reúnen datos y los analizan con detenimiento, a partir de múltiples y posibles alternativas. Además, observan en detalle el actuar propio y el de los demás y, no intervienen hasta que poseen un panorama amplio de la situación de aprendizaje en la que se encuentran.

\section{Discusión}

A partir de los resultados que han permitido una caracterización de la composición de estilos de aprendizaje, de los estudiantes de primer año de la Facultad de Ciencias Médicas, se propone una guía metodológica docente para la enseñanza de la Física mediante el uso de recursos de Tecnologías de la Información y la Comunicación TIC, según Estilos de Aprendizaje del Modelo Honey-Alonso. Se parte del peso porcentual 
de cada estilo, para diseñar de manera correspondiente, una cantidad relativa de actividades afines a dicho estilo de aprendizaje. A continuación, se muestra una tabla que especifica la proporción utilizada para el desarrollo de las actividades de las Unidades de la Guía propuesta.

\begin{tabular}{|c|c|c|}
\hline Estilo aprendizaje & $\begin{array}{c}\text { \% estilos de estilos } \\
\text { aprendizaje }\end{array}$ & $\begin{array}{c}\text { Cantidad de } \\
\text { actividades }\end{array}$ \\
\hline Activo & $10 \%$ & 1 \\
\hline Pragmático & $17 \%$ & 2 \\
\hline Teórico & $18 \%$ & 2 \\
\hline Reflexivo & $55 \%$ & 5 \\
\hline
\end{tabular}

Tabla 2. Porcentaje de Estilos de Aprendizaje

Nota. Esta tabla muestra los diferentes estilos de aprendizaje de los estudiantes de primer año de la Facultad de Ciencias Médicas USAC.

Dada la mayoría de los estudiantes, con un estilo reflexivo, dentro de la composición de primer año de la Facultad de Ciencias Médicas, es importante considerar que propio del estilo reflexivo de aprendizaje, se consideren aspectos que destaquen el fomento de criterios científicos, que incluyen el análisis, la observación y el razonamiento formal, y el estudio de escenarios para tomar una decisión.

Junto con la mejora de los procesos didácticos para la enseñanza de las ciencias, como lo es el caso de la Física, es importante, gestionar una mejora de los recursos educativos de tipo tecnológico disponibles en la Facultad de Ciencias Médicas: disponibilidad de internet de alta velocidad, uso de aulas virtuales con el acompañamiento de procesos de actualización docente, fomento de la innovación en tecnología educativa.

\section{Agradecimiento}

Al maestro Ludwing Antonio Llamas Álvarez de la Facultad de Humanidades de la Universidad de San Carlos de Guatemala por su apoyo en la realización de la investigación. 


\section{Referencias}

Canos, M. y Líen, C. (2015). El uso de las nuevas tecnologías aplicadas a la educación superior. XVII Jornadas ASEPUMA - V Encuentro Internacional Rect@ Vol Actas_17, Issue 1: 612. Disponible en: https://www.researchgate.net/publication/26626090_El_uso_de_ las_nuevas_tecnologias_aplicadas_a_la_educacion_superior.

Escurra, L. (2011). Análisis psicométrico del Cuestionario de Honey y Alonso de Estilos de Aprendizaje (CHAEA) con los modelos de la Teoría Clásica de los Test y de Rasch. Persona, (14),71-109. ISSN: 1560-6139. Disponible en: https://www.redalyc.org/articulo. oa?id=1471/147122650003. doi: https://doi.org/10.26439/ persona2011.n014.253

Galarza, M. T. (2015). La Autoevaluación de los Procesos de enseñanza Y aprendizaje como mediación para la Innovación de los ambientes escolares. [Tesis de Especialización en Docencia Universitaria, Universidad Libre Bogotá D.C.] http://repository.unilibre.edu.co/ bitstream/handle/10901/8299/LA\%20AUTO\%20EVALUACION\%20 DE\%20LOS\%20PROCESOS\%20DE\%20ENSE\%C3\%91ANZA. pdf?sequence $=1$

García, J. (2004). Estudios descriptivos. Investigación NURE. Disponible en: http://webpersonal.uma.es/ jmpaez/websci/bloqueiii/docbiii/ estudios\%20descriptivos.pdf

Molina, A. (2012). Las TIC en la Educación Superior como vía de formación y desarrollo competencial en la Sociedad del Conocimiento. Electrónica de investigación Docencia Creativa. [Archivo PDF]. http://www.ugr.es/ miguelgr/ReiDoCrea-Vol.1-Art.15-Molina.pdf

Ríos, R. (2014). Factores predisponentes a la deserción en primer año de la carrera de Medicina. Facultad de Ciencias Médicas de la Universidad de San Carlos de Guatemala. IV CLAVES. [Archivo PDF]. https://digi. usac.edu.gt/bvirtual/informes/puie/INF-2014-32.pdf

Téliz, F. (2015). Uso didáctico de las TIC en las buenas prácticas de enseñanza de las matemáticas: Estudio de las opiniones y concepciones de docentes de educación secundaria en el departamento de Artigas. Cuadernos de Investigación Educativa, 6(2), 13-31. Recuperado en 26 de octubre de 2020, de http://www.scielo.edu.uy/scielo. php?script=sci_arttext\&pid=S1688-93042015000200002\&Ing=es\&t| ng=es. doi: https://doi.org/10.18861/cied.2015.6.2.34 


\section{Sobre el autor}

Profesional de Ingeniería especialidad en Mecánica Industrial, pensum cerrado de la Escuela de Estudios de Postgrado de la Facultad de Humanidades con Especialidad en Educación de la Maestría en Docencia Universitaria, Universidad de San Carlos de Guatemala, y Diplomado tutor virtual en el programa Rad de la Universidad de San Carlos de Guatemala.

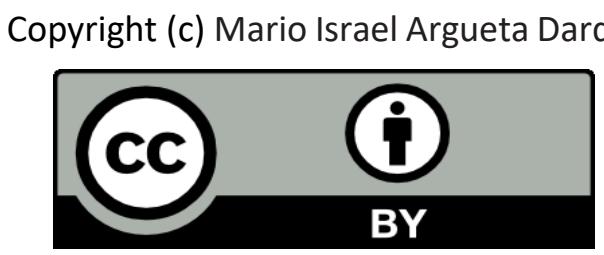

Este texto está protegido por una licencia CreativeCommons 4.0.

Usted es libre para compartir, copiar y redistribuir el material en cualquier medio o formato y adaptar el documento, remezclar, transformar y crear a partir del material para cualquier propósito, incluso comercialmente, siempre que cumpla la condición de atribución: usted debe reconocer el crédito de una obra de manera adecuada, proporcionar un enlace a la licencia, e indicar si se han realizado cambios. Puede hacerlo en cualquier forma razonable, pero no de forma tal que sugiera que tiene el apoyo del licenciante o lo recibe por el uso que hace. 